\title{
On the product of heights of algebraic numbers summing to real numbers
}

\author{
by
}
J. Garza (Brownsville, TX), M. I. M. IshaK (Manhattan, KS) and C. Pinner (Manhattan, KS)

1. Introduction. Amongst the absolute values in a place $v$ of an algebraic number field $\mathbb{K}$, two play a role in this article. If $v$ is archimedean, let $\|\cdot\|_{v}$ denote the unique absolute value in $v$ that restricts to the usual archimedean absolute value on $\mathbb{Q}$. If $v$ is non-archimedean and $v \mid p$, let $\|\cdot\|_{v}$ denote the unique absolute value in $v$ that restricts to the usual $p$-adic absolute value on $\mathbb{Q}$. For each place $v$ of $\mathbb{K}$, let $\mathbb{K}_{v}$ and $\mathbb{Q}_{v}$ be the completions of $\mathbb{K}$ and $\mathbb{Q}$ with respect to $v$ and define the local degree of $v$ as $d_{v}=\left[\mathbb{K}_{v}: \mathbb{Q}_{v}\right]$. For all places $v$ let $|\cdot|_{v}=\|\cdot\|_{v}^{d_{v} / d}$.

The absolute values $|\cdot|_{v}$ satisfy the product rule: if $\alpha \in \mathbb{K}^{\times}$, then $\prod_{v}|\alpha|_{v}=1$. The absolute (logarithmic) Weil height of $\alpha$ is defined as $h(\alpha)=\sum_{v} \log ^{+}|\alpha|_{v}$ where the sum is over all places $v$ of $\mathbb{K}$. Because of the way in which the absolute values $|\cdot|_{v}$ are normalized, $h(\alpha)$ does not depend on the field $\mathbb{K}$ in which $\alpha$ is contained. If $\alpha_{i}$ and $\alpha_{j}$ are Galois conjugates then $h\left(\alpha_{i}\right)=h\left(\alpha_{j}\right)$ and for $\alpha \in \overline{\mathbb{Q}}^{\times}, h(\alpha)=h\left(\alpha^{-1}\right)$.

By Kronecker's theorem $h(\alpha)=0$ if and only if $\alpha=0$ or $\alpha \in \operatorname{Tor}\left(\overline{\mathbb{Q}}^{\times}\right)$. In 1933, Lehmer [5] asked whether or not there exists a constant $\varrho>1$ such that

$$
\operatorname{deg}(\alpha) h(\alpha) \geq \log \varrho
$$

in all other cases. For algebraic numbers $\alpha$ the Mahler measure $\operatorname{M}(\alpha)$ of $\alpha$ is defined by $\operatorname{deg}(\alpha) h(\alpha)=\log \mathrm{M}(\alpha)$. The smallest non-zero Mahler measure known is that of the roots of $x^{10}+x^{9}-x^{7}-x^{6}-x^{5}-x^{4}-x^{3}+x+1$, and it is thought by many that if the answer to Lehmer's question is yes then the minimum possible $\varrho$ is the log of unique real root larger than one of the above polynomial.

2010 Mathematics Subject Classification: Primary 11R09; Secondary 11G50.

Key words and phrases: Mahler measure, Weil height. 
Lehmer's question remains unresolved to this day though affirmative answers have been provided in some special cases. Schinzel [7] has shown that for $\alpha$ totally real we may take $\varrho=\frac{1}{2} \log ((1+\sqrt{5}) / 2) \cdot \operatorname{deg}(\alpha)$ on the right side of (1.1). Höhn and Skoruppa [4] used a different proof to establish the same result. Zhang [9] showed that there exists $\varrho>1$ such that

$$
h(\alpha)+h(1-\alpha) \geq \log \varrho
$$

for $\alpha$ different from 0,1 and the primitive sixth roots of unity. Zagier [8] was able to establish $\log \varrho=\frac{1}{2} \log ((1+\sqrt{5}) / 2)$ as a minimum for the right side of (1.2) with cases of equality identified. As Zagier notes, it is interesting that this is the same lower bound that appears in Schinzel's lower bound for the height of totally real algebraic numbers. Beukers and Zagier [1] generalized the results of [8] with the following: if $\alpha_{1}, \ldots, \alpha_{r} \in \overline{\mathbb{Q}}^{\times}$are such that

$$
\alpha_{1}+\cdots+\alpha_{r}=N \neq \alpha_{1}^{-1}+\cdots+\alpha_{r}^{-1}
$$

where $N$ is a rational integer, then

$$
h\left(\alpha_{1}\right)+\cdots+h\left(\alpha_{r}\right) \geq \frac{1}{2} \log \left(\frac{1+\sqrt{5}}{2}\right) .
$$

More recently, Samuels [6] extended this last result by allowing the $N$ in (1.3) to be any totally real algebraic integer. In the case $r=1$, Samuels' result captures Schinzel's lower bound for the height of totally real algebraic integers.

After Samuels, and in a different direction, Garza [2] established the following result: Let $\alpha$ be an algebraic number different from 0 and \pm 1 . Let $\mathcal{R}_{\alpha}$ be the proportion of the conjugates of $\alpha$ that are real. Then

$$
h(\alpha) \geq \frac{\mathcal{R}_{\alpha}}{2} \log \left(\frac{2^{1-1 / \mathcal{R}_{\alpha}}+\sqrt{4^{1-1 / \mathcal{R}_{\alpha}}+4}}{2}\right) .
$$

In the case $\mathcal{R}_{\alpha}=1$, formula (1.5) also recaptures Schinzel's lower bound for the height of totally real algebraic numbers. Following [2], Höhn [3] showed that his proof of [4] could be extended to also establish (1.5) in the case that $\alpha$ is an algebraic integer. Our goal is to show that the stated results of [2] and [6] are in fact consequences of an even more general formula. In particular, we generalize the method of [3] to establish the following:

Theorem 1. Let $\alpha_{1}, \ldots, \alpha_{r} \in \overline{\mathbb{Q}}^{\times}$be such that $\alpha_{1}+\cdots+\alpha_{r} \neq \alpha_{1}^{-1}+$ $\cdots+\alpha_{r}^{-1}$. Let $\mathcal{R}_{\mathcal{S}}$ be the proportion of the conjugates of $\mathcal{S}=\alpha_{1}+\cdots+\alpha_{r}$ that are real. Then

$$
\sum_{i=1}^{r} h\left(\alpha_{i}\right) \geq \frac{\mathcal{R}_{\mathcal{S}}}{2} \log \left(\frac{(2 r)^{1-1 / \mathcal{R}_{\mathcal{S}}}+\sqrt{(2 r)^{2\left(1-1 / \mathcal{R}_{\S}\right)}+4}}{2}\right) .
$$


In the case $\mathcal{R}_{\mathcal{S}}=1$ we not only recover the result of Samuels [6] but are able to eliminate the requirement that $\mathcal{S}$ be an integer. In the case $r=1$ we recover Theorem 1 of Garza [2]. It is natural to ask whether a generalization of Theorem 2 of Garza [2] can also be found. The answer is provided by the following.

TheOREM 2. Let $\alpha_{1}, \ldots, \alpha_{r} \in \overline{\mathbb{Q}}^{\times}$be such that $\alpha_{1}+\cdots+\alpha_{r} \neq \bar{\alpha}_{1}^{-1}+$ $+\cdots+\bar{\alpha}_{r}^{-1}$. Let $\mathbb{K}$ be the Galois closure of $\mathbb{Q}\left(\alpha_{1}+\cdots+\alpha_{r}\right)$ and let $\mathrm{G}=$ $\operatorname{Aut}(\mathbb{K} / \mathbb{Q})$. Let $\eta: \mathbb{K} \hookrightarrow \mathbb{C}$ be an embedding and let $\xi \in \mathrm{G}$ correspond to complex conjugation with respect to $\eta$. Let $C_{\mathrm{G}}(\xi)=\{\sigma \in \mathrm{G}: \xi \sigma=\sigma \xi\}$ and let $n=\left[\mathrm{G}: C_{\mathrm{G}}(\xi)\right]$. Then

$$
\sum_{i=1}^{r} h\left(\alpha_{i}\right) \geq \frac{1}{2 n} \log \left(\frac{(2 r)^{1-n}+\sqrt{(2 r)^{2(1-n)}+4}}{2}\right) .
$$

2. Preliminary lemmas. In this section we establish Lemmas 1 and 2, which will be used to prove Theorems 1 and 2 .

Lemma 1. For $0<a<1 / 2$ and real $x_{i}>0$

$$
\begin{aligned}
f\left(x_{1}, \ldots, x_{r}\right) & =\frac{\left(\sum_{i=1}^{r}\left|x_{i}-x_{i}^{-1}\right|\right)^{a}}{\prod_{i=1}^{r} \max \left\{1,\left|x_{i}\right|\right\}^{1 / 2} \max \left\{1,\left|x_{i}^{-1}\right|\right\}^{1 / 2}} \\
& \leq \frac{(4 a)^{a}(1-2 a)^{(1-2 a) / 4}}{(1+2 a)^{(1+2 a) / 4}} .
\end{aligned}
$$

Proof. By symmetry we may assume that each $x_{i} \geq 1$. In this case,

$$
f\left(x_{1}, \ldots, x_{r}\right)=\frac{\left(\sum_{i=1}^{r}\left(x_{i}-x_{i}^{-1}\right)\right)^{a}}{\prod_{i=1}^{r} x_{i}^{1 / 2}} .
$$

If one of the $x_{i}=1$ then the function reduces to $r-1$ variables. We can thus suppose that each $x_{i}>1$.

For $i=1, \ldots, r$

$$
\frac{\partial f}{\partial x_{i}}=\frac{\left(\sum_{j=1}^{r}\left(x_{j}-x_{j}^{-1}\right)\right)^{a}}{\prod_{j=1}^{r} x_{j}^{1 / 2}}\left\{\frac{a\left(1+1 / x_{i}^{2}\right)}{\sum_{j=1}^{r}\left(x_{j}-x_{j}^{-1}\right)}-\frac{1}{2 x_{i}}\right\}
$$

and $\partial f / \partial x_{i}=0$ is equivalent to

$$
\sum_{j=1}^{r}\left(x_{j}-x_{j}^{-1}\right)=2 a\left(x_{i}+x_{i}^{-1}\right) .
$$

It follows that if $\partial f / \partial x_{1}=\cdots=\partial f / \partial x_{r}=0$, then for all $i$ and $j$,

$$
x_{i}+x_{i}^{-1}=x_{j}+x_{j}^{-1} \text {. }
$$


Since each $x_{i}>1$ it follows that $x_{1}=\cdots=x_{r}$. We let $x$ be this common value. Equality (2.1) thus reduces to

$$
r\left(x-x^{-1}\right)=2 a\left(x+x^{-1}\right) .
$$

Solving this last equation for $x$ results in $x=\sqrt{(r+2 a) /(r-2 a)}$. Let $\vec{x}=$ $(x, \ldots, x)$. Then

$$
f(\vec{x})=\frac{r^{a}\left(x-x^{-1}\right)^{a}}{x^{r / 2}}=\frac{r^{a}(4 a)^{a}(r-2 a)^{(r-2 a) / 4}}{(r+2 a)^{(r+2 a) / 4}} .
$$

We wish to show that the expression on the right hand side of the last equation is a decreasing function of $r$. For this purpose define the function $g(r)$ as follows:

$$
g(r)=\frac{r^{a}(r-2 a)^{(r-2 a) / 4}}{(r+2 a)^{(r+2 a) / 4}} .
$$

Then

$$
\ln g(r)=a \ln r+\frac{1}{4}(r-2 a) \ln (r-2 a)-\frac{1}{4}(r+2 a) \ln (r+2 a)
$$

and so

$$
\begin{aligned}
\frac{d}{d r}[\ln g(r)] & =\frac{a}{r}-\frac{1}{4} \ln \left(\frac{r+2 a}{r-2 a}\right)=\frac{a}{r}-\frac{1}{4} \ln \left(\frac{1+2 a / r}{1-2 a / r}\right) \\
& =\frac{a}{r}-\frac{1}{4}\left(-\sum_{j=1}^{\infty}(-1)^{j}(2 a / r)^{j}+\sum_{j=1}^{\infty}(2 a / r)^{j}\right) \\
& =\frac{a}{r}-\frac{1}{2} \sum_{j \text { odd }}(2 a / r)^{j}=-\frac{1}{2} \sum_{j \geq 3, \text { odd }}(2 a / r)^{j}<0 .
\end{aligned}
$$

Since $g(r)$ is a decreasing function of $r$ it follows that $f\left(x_{1}, \ldots, x_{r}\right) \leq$ $(4 a)^{a} g(1)$.

Lemma 2. Given $r \in \mathbb{N}$ and $0<\beta<1$ define the function

$$
P(a)=(2 r)^{a(1-\beta)}\left(\frac{(4 a)^{a}(1-2 a)^{(1-2 a) / 4}}{(1+2 a)^{(1+2 a) / 4}}\right)^{\beta}
$$

and $0<a^{\prime}<1 / 2$ by

$$
a^{\prime}=\frac{1}{2 \sqrt{1+4^{1 / \beta} r^{2(1 / \beta-1)}}} .
$$

Then $P$ attains its minimum on $0<a<1 / 2$ at $a^{\prime}$ and

$$
P\left(a^{\prime}\right)=\left(\sqrt{1+\left(2^{-1 / \beta} r^{1-1 / \beta}\right)^{2}}+2^{-1 / \beta} r^{1-1 / \beta}\right)^{-\beta / 2} .
$$


Proof. We first note that $P(a)$ is continuously differentiable on the interval $(0,1 / 2)$ and that

$$
\lim _{a \rightarrow 0} P(a)=1 \quad \text { and } \quad \lim _{a \rightarrow 1 / 2} P(a)>1 .
$$

We proceed by noting that if $u=2 a$ then $P(a)=F(u)^{-1}$ where

$$
F(u)=\frac{(1+u)^{\beta(1+u) / 4}}{(2 r)^{u(1-\beta) / 2}(2 u)^{u \beta / 2}(1-u)^{(1-u) \beta / 4}} .
$$

Then

$$
\begin{aligned}
(F(u))^{2 / \beta}= & \frac{(1+u)^{(1+u) / 2}}{(2 r)^{u(1 / \beta-1)}(2 u)^{u}(1-u)^{(1-u) / 2}}, \\
\frac{2}{\beta} \ln F(u)= & \frac{1}{2}(1+u) \ln (1+u)-u\left(\frac{1}{\beta}-1\right) \ln 2 r \\
& -u \ln 2 u-\frac{1}{2}(1-u) \ln (1-u), \\
\frac{2}{\beta} \frac{d}{d u} \ln F(u)= & \frac{1}{2}+\frac{1}{2} \ln (u+1)-\left(\frac{1}{\beta}-1\right) \ln 2 r \\
& -\ln 2 u-1+\frac{1}{2}+\frac{1}{2} \ln (1-u) \\
= & \frac{1}{2} \ln (u+1)-\left(\frac{1}{\beta}-1\right) \ln 2 r-\ln 2 u+\frac{1}{2} \ln (1-u) \\
= & \frac{1}{2} \ln \left(\frac{1-u^{2}}{4 u^{2}}\right)-\left(\frac{1}{\beta}-1\right) \ln 2 r .
\end{aligned}
$$

The equation

$$
\frac{d}{d u} \ln F(u)=0
$$

is thus equivalent to

$$
\ln \left(\frac{1-u^{2}}{4 u^{2}}\right)=\ln \left(\left(4 r^{2}\right)^{1 / \beta-1}\right),
$$

which has the unique solution

$$
u=\frac{1}{\sqrt{1+4^{1 / \beta} r^{2(1 / \beta-1)}}}
$$

on the interval $(0,1)$. From this we have

$$
a=\frac{1}{2} \cdot \frac{1}{\sqrt{1+4^{1 / \beta} r^{2(1 / \beta-1)}}}<\frac{1}{2} .
$$

Since this is the value of $u$ maximizing $\ln F(u)$ and hence $F(u)$ and since 
$P(a)=F(u)^{-1}$, we have found the value of $a$ minimizing $P(a)$. So we write

$$
a^{\prime}=\frac{1}{2} \cdot \frac{1}{\sqrt{1+4^{1 / \beta} r^{2(1 / \beta-1)}}}<\frac{1}{2} .
$$

We now substitute $2 a^{\prime}$ into $F(u)$ and obtain the maximum

$$
F\left(2 a^{\prime}\right)=\left(\sqrt{1+\left(2^{-1 / \beta} r^{1-1 / \beta}\right)^{2}}+2^{-1 / \beta} r^{1-1 / \beta}\right)^{\beta / 2} .
$$

3. Proofs of Theorems 1 and 2. Let

$$
a=\frac{1}{2 \sqrt{1+4^{1 / \beta} r^{2(1 / \beta-1)}}} .
$$

Let $\mathcal{S}=\alpha_{1}+\cdots+\alpha_{r}, \mathcal{S}_{-1}=\alpha_{1}^{-1}+\cdots+\alpha_{r}^{-1}$, and $E=\overline{\mathcal{S}}-\mathcal{S}_{-1}$. Let $\mathbb{F}$ be the Galois closure of $\mathbb{Q}\left(\alpha_{1}, \ldots, \alpha_{r}\right)$, let $\mathrm{H} \equiv \operatorname{Aut}(\mathbb{F} / \mathbb{Q})$ and extend $\eta: \mathbb{F} \hookrightarrow \mathbb{C}$. Let $\xi_{\mathbb{F}} \in \mathrm{H}$ correspond to complex conjugation with respect to $\eta$. Let $\mathrm{H}_{\mathbb{K}}$ be the subgroup of $\mathrm{H}$ fixing the field $\mathbb{K}$. Then $\mathrm{H}_{\mathbb{K}} \unlhd \mathrm{H}$. Let $\mathcal{A}=\{\sigma \in \mathrm{H}: \sigma(\overline{\mathcal{S}})=\overline{\sigma(\mathcal{S})}\}$ and note that $\sigma \in \mathcal{A}$ if and only if $\xi_{\mathbb{F}} \sigma \in \mathcal{A}$. Let $\phi: \mathrm{H} \rightarrow \mathrm{H} / \mathrm{H}_{\mathbb{K}}$ be the natural projection homomorphism. Suppose that $\omega \in \mathrm{H}$ is such that $\phi(\omega) \in C_{\mathrm{G}}(\xi) \mathrm{H}_{\mathbb{K}}$. Then $\phi^{-1}(\phi(\omega)) \subseteq \mathcal{A}$. Moreover, if $\omega$, $\tau \in \mathrm{G}$ and $\omega \neq \tau$ then $\phi^{-1}(\omega) \cap \phi^{-1}(\tau)=\emptyset$. It follows that $\left|\mathrm{H}_{\mathbb{K}}\right| \cdot\left|C_{\mathrm{G}}(\xi)\right|$ $\leq|\mathcal{A}|$ and that $n \geq|\mathrm{H}| /|\mathcal{A}|$. For $\sigma \in \mathcal{A}$ we have

$$
\sigma(E)=\sum_{i=1}^{r}\left(\overline{\sigma\left(\alpha_{i}\right)}-\frac{1}{\sigma\left(\alpha_{i}\right)}\right) .
$$

Since $E \neq 0$ it follows from the product rule that

$$
\prod_{v}|E|_{v}^{a}=1
$$

where the product is over all places $v$ of $\mathbb{F}$. From the ultrametric inequality, for $v \nmid \infty$ it follows that

$$
|E|_{v}^{a} \leq \prod_{i=1}^{r} \max \left\{1,\left|\alpha_{i}\right|_{v}\right\}^{a} \prod_{i=1}^{r} \max \left\{1,\left|\alpha_{i}\right|_{v}^{-1}\right\}^{a} .
$$

Since $\mathbb{F}$ is Galois, it follows that all local archimedean degrees are equal and we let $d_{\infty}$ be the common archimedean local degree. We now define the archimedean absolute value $|\cdot|_{\eta}$ on $\mathbb{F}$ by $|\lambda|_{\eta}=\|\eta(\lambda)\|_{\infty}^{d_{\infty} / d}$ for $\lambda \in \mathbb{F}$. From the Galois action on places it follows that for each archimedean place $v$ of $\mathbb{F}$ there exists $\sigma \in \mathrm{H}$ such that $|\lambda|_{v}=\|\eta(\sigma(\lambda))\|_{\eta}^{d_{\infty} / d}$ for $\lambda \in \mathbb{F}$. In this case we write $|\cdot|_{v}=|\cdot|_{\sigma}$ and note that $|\cdot|_{\sigma}=|\cdot|_{\xi \sigma}$. For $\sigma \notin \mathcal{A}$ we have

$$
|E|_{\sigma}^{a} \leq(2 r)^{a d_{\infty} / d} \prod_{i=1}^{r} \max \left\{1,\left|\alpha_{i}\right|_{\sigma}\right\}^{a} \prod_{i=1}^{r} \max \left\{1,\left|\alpha_{i}\right|_{\sigma}^{-1}\right\}^{a} .
$$


For $\sigma \in \mathcal{A}$ define $\sigma\left(\alpha_{i}\right)^{\prime}=\sigma\left(\alpha_{i}\right)$ for $\left\|\alpha_{i}\right\|_{\sigma} \geq 1$ and $\sigma\left(\alpha_{i}\right)^{\prime}=\sigma\left(\alpha_{i}\right)^{-1}$ for $\left\|\alpha_{i}\right\|_{\sigma}<1$. For $\sigma \in \mathcal{A}$ we have

$$
\begin{aligned}
|E|_{\sigma}^{a} & \left.\leq \| \sum_{i=1}^{r} \overline{\sigma\left(\alpha_{i}\right.}\right)-\frac{1}{\sigma\left(\alpha_{i}\right)} \|_{\eta}^{a d_{\infty} / d} \leq\left(\sum_{i=1}^{r}\left\|\overline{\sigma\left(\alpha_{i}\right)}-\frac{1}{\sigma\left(\alpha_{i}\right)}\right\|_{\eta}\right)^{a d_{\infty} / d} \\
& \leq\left(\sum_{i=1}^{r}\left\|\overline{\sigma\left(\alpha_{i}\right)^{\prime}}\right\|_{\eta}-\frac{1}{\left\|\sigma\left(\alpha_{i}\right)^{\prime}\right\|_{\eta}}\right)^{a d_{\infty} / d}
\end{aligned}
$$

and from Lemma 1,

$$
\begin{aligned}
|E|_{\sigma}^{a} & \leq\left(\frac{(4 a)^{a}(1-2 a)^{(1-2 a) / 4}}{(1+2 a)^{(1+2 a) / 4}} \cdot \prod_{i=1}^{r} \max \left\{1,\left\|\sigma\left(\alpha_{i}\right)^{\prime}\right\|_{\eta}\right\}^{1 / 2}\right)^{d_{\infty} / d} \\
& \leq\left(\frac{(4 a)^{a}(1-2 a)^{(1-2 a) / 4}}{(1+2 a)^{(1+2 a) / 4}}\right)^{d_{\infty} / d} \cdot \prod_{i=1}^{r} \max \left\{1,\left|\sigma\left(\alpha_{i}\right)^{\prime}\right|_{\eta}\right\}^{1 / 2} .
\end{aligned}
$$

Taking the product over $\mathcal{A}$, we get

$$
\prod_{\sigma \in \mathcal{A}}|E|_{\sigma}^{a} \leq\left(\frac{(4 a)^{a}(1-2 a)^{(1-2 a) / 4}}{(1+2 a)^{(1+2 a) / 4}}\right)^{d_{\infty}|\mathcal{A}| /|\mathrm{H}|}\left(\prod_{\sigma \in \mathcal{A}} \prod_{i=1}^{r} \max \left\{1,\left|\sigma\left(\alpha_{i}\right)^{\prime}\right|_{\eta}\right\}^{1 / 2}\right) .
$$

Using the identity $\sum d_{v} / d=1$ and taking the product over $\sigma \notin \mathcal{A}$ we have

$$
\prod_{\sigma \notin \mathcal{A}}|E|_{\sigma}^{a} \leq\left((2 r)^{a d_{\infty}(1-|\mathcal{A}| /|\mathrm{H}|)}\right)\left(\prod_{\sigma \notin \mathcal{A}} \prod_{i=1}^{r} \max \left\{1,\left|\sigma\left(\alpha_{i}\right)^{\prime}\right|_{\eta}\right\}^{a}\right) .
$$

From $0<a<1 / 2$, the product rule, the Galois action on places, $\xi \mathcal{A}=\mathcal{A}$ and $h(\alpha)=h\left(\alpha^{-1}\right)$ we have

$$
1 \leq(2 r)^{a(1-|\mathcal{A}| /|\mathrm{H}|)}\left(\frac{(4 a)^{a}(1-2 a)^{(1-2 a) / 4}}{(1+2 a)^{(1+2 a) / 4}}\right)^{|\mathcal{A}| /|\mathrm{H}|} \prod_{i=1}^{r} e^{h\left(\alpha_{i}\right)} .
$$

Since $n \geq|\mathrm{H}| /|\mathcal{A}|$ and $0<a<1 / 2$ it follows that

$$
1 \leq(2 r)^{a(1-1 / n)}\left(\frac{(4 a)^{a}(1-2 a)^{(1-2 a) / 4}}{(1+2 a)^{(1+2 a) / 4}}\right)^{1 / n} \prod_{i=1}^{r} e^{h\left(\alpha_{i}\right)} .
$$

From Lemma 2 with $\beta=1 / n$ we have

$$
\left(\sqrt{1+\left(2^{-n} r^{1-n}\right)^{2}}+2^{-n} r^{1-n}\right)^{1 / 2 n} \leq \prod_{i=1}^{r} e^{h\left(\alpha_{i}\right)},
$$

which is equivalent to

$$
\sum_{i=1}^{r} h\left(\alpha_{i}\right) \geq \frac{1}{2 n} \log \left(\frac{(2 r)^{1-n}+\sqrt{4+4^{1-n} r^{2(1-n)}}}{2}\right) .
$$

This completes the proof of Theorem 2 . 
For the proof of Theorem 1, we consider $\mathcal{S}-\mathcal{S}_{-1}$. We again let $\mathbb{F}$ be the Galois closure of $\mathbb{Q}\left(\alpha_{1}, \ldots, \alpha_{r}\right)$ and let $\mathrm{G}=\operatorname{Aut}(\mathbb{F} / \mathbb{Q})$. For $\sigma \in \mathrm{G}$ such that $\sigma(\mathcal{S})$ is real we have

$$
\begin{aligned}
\sigma\left(\mathcal{S}-\mathcal{S}_{-1}\right) & =\sigma(\mathcal{S})-\sigma\left(\mathcal{S}_{-1}\right)=\overline{\sigma(\mathcal{S})}-\sigma\left(\mathcal{S}_{-1}\right) \\
& =\overline{\sigma\left(\alpha_{1}\right)}+\cdots+\overline{\sigma\left(\alpha_{r}\right)}-\frac{1}{\sigma\left(\alpha_{1}\right)}-\cdots-\frac{1}{\sigma\left(\alpha_{r}\right)} \\
& =\sum_{i=1}^{r}\left(\overline{\sigma\left(\alpha_{i}\right)}-\frac{1}{\sigma\left(\alpha_{i}\right)}\right)
\end{aligned}
$$

so that proof of Theorem 1 follows from the proof of Theorem 2 but with $E$ replaced by $\mathcal{S}-\mathcal{S}_{-1}$.

\section{References}

[1] F. Beukers and D. Zagier, Lower bounds of heights of points on hypersurfaces, Acta Arith. 79 (1997), 103-111.

[2] J. Garza, On the height of algebraic numbers with real conjugates, ibid. 128 (2007), 385-389.

[3] G. Höhn, On a theorem of Garza regarding algebraic integers with real conjugates, preprint.

[4] G. Höhn and N. P. Skoruppa, Un résultat de Schinzel, J. Théorie Nombres Bordeaux 5 (1993), 185.

[5] D. H. Lehmer, Factorization of certain cyclotomic functions, Ann. of Math. 34 (1933), 461-479.

[6] C. L. Samuels, Lower bounds on the projective heights of algebraic points, Acta Arith. 125 (2006), 41-50.

[7] A. Schinzel, On the product of the conjugates outside the unit circle of an algebraic number, ibid. 24 (1973), 385-399; Addendum, ibid. 26 (1975), 329-331.

[8] D. Zagier, Algebraic numbers close to both 0 and 1, Math. Comp. 61 (1993), 485-491.

[9] S. Zhang, Positive line bundles on arithmetic surfaces, Ann. of Math. 136 (1992), $569-587$.

J. Garza

Department of Mathematics

The University of Texas at Brownsville

80 Fort Brown

Brownsville, TX 78520, U.S.A.

E-mail: John.Garza@utb.edu
M. I. M. Ishak, C. Pinner

Department of Mathematics

Kansas State University Manhattan, KS 66506, U.S.A. E-mail: mimishak@math.ksu.edu pinner@math.ksu.edu

Received on 5.1.2009

and in revised form on 23.7.2009 